May 2006

\title{
A randomized trial of a multicomponent home intervention to reduce functional difficulties in older adults
}

\author{
Laura N. Gitlin \\ Thomas Jefferson University \\ Laraine Winter \\ Thomas Jefferson University \\ Marie P. Dennis \\ Thomas Jefferson University \\ Mary Corcoran \\ George Washington University \\ Walter W. Hauck

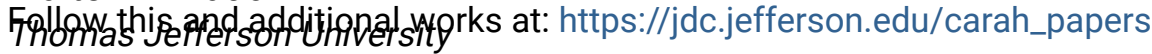 \\ Part of the Behavior and Behavior Mechanisms Commons \\ Let us know how access to this document benefits you
}

\section{Recommended Citation}

Gitlin, Laura N.; Winter, Laraine; Dennis, Marie P.; Corcoran, Mary ; and Hauck, Walter W., "A randomized trial of a multicomponent home intervention to reduce functional difficulties in older adults" (2006). Center for Applied Research on Aging and Health Research Papers. Paper 5. https://jdc.jefferson.edu/carah_papers/5

This Article is brought to you for free and open access by the Jefferson Digital Commons. The Jefferson Digital Commons is a service of Thomas Jefferson University's Center for Teaching and Learning (CTL). The Commons is a showcase for Jefferson books and journals, peer-reviewed scholarly publications, unique historical collections from the University archives, and teaching tools. The Jefferson Digital Commons allows researchers and interested readers anywhere in the world to learn about and keep up to date with Jefferson scholarship. This article has been accepted for inclusion in Center for Applied Research on Aging and Health Research Papers by an authorized administrator of the Jefferson Digital Commons. For more information, please contact: JeffersonDigitalCommons@jefferson.edu. 


\title{
A Randomized Trial of a Multicomponent Home Intervention to Reduce Functional Difficulties in Older Adults
}

Laura N. Gitlin, $\mathrm{PhD},{ }^{1}$ Laraine Winter, $\mathrm{PhD}{ }^{1}$ Marie P. Dennis, PhD, EdM, ${ }^{1}$ Mary Corcoran, $\mathrm{PhD}, \mathrm{OTR} / \mathrm{L}^{2}$ Sandy Schinfeld, $\mathrm{MPH},{ }^{1}$ and Walter W. Hauck, $\mathrm{PhD}{ }^{3}$

From the ${ }^{1}$ Center for Applied Research on Aging and Health, ${ }^{2}$ Department of Pharmacology and Experimental Therapeutics, Division of Biostatistics, Thomas Jefferson University, Philadelphia, Pennsylvania; and ${ }^{3}$ Department of Health Care Sciences, The George Washington University, Washington, DC.

Address correspondence to Laura N. Gitlin, PhD, Center for Applied Research on Aging and Health, Thomas Jefferson University, 130 S 9th Street, Suite 513, Philadelphia, PA 19130. E-mail: laura.gitlin@jefferson.edu

\begin{abstract}
:
OBJECTIVES: To test the efficacy of a multicomponent intervention to reduce functional difficulties, fear of falling, and home hazards and enhance self-efficacy and adaptive coping in older adults with chronic conditions.
\end{abstract}

DESIGN: A prospective, two-group, randomized trial. Participants were randomized to a treatment group or no-treatment group.

SETTING: Urban community-living older people. PARTICIPANTS: Three hundred nineteen community-living adults aged 70 and older who reported difficulty with one or more activities of daily living.

INTERVENTION: Occupational and physical therapy sessions involving home 
modifications and training in their use; instruction in strategies of problem-solving, energy conservation, safe performance, and fall recovery techniques; and balance and muscle strength training.

MEASUREMENTS: Outcome measures included self-rated functional difficulties with ambulation, instrumental activities of daily living, activities of daily living, fear of falling, confidence performing daily tasks, and use of adaptive strategies. Observations of home hazards were also conducted.

RESULTS: At 6 months, intervention participants had less difficulty than controls with instrumental activities of daily living $(P=.04,95 \%$ confidence interval $(C I)=-0.28-$ $0.00)$ and activities of daily living $(P=.03,95 \% C I=-0.24$ to -0.01$)$, with largest reductions in bathing $(P=.02,95 \% C I=-0.52$ to -0.06$)$ and toileting $(P=.049$, $95 \% C I=-0.35-0.00)$. They also had greater self-efficacy $(P=.03,95 \% C I=0.02$ $-0.27)$, less fear of falling $(P=.001,95 \% C I=0.26-0.96)$, fewer home hazards $(P$ $=.05,95 \% \mathrm{CI}=-3.06-0.00)$, and greater use of adaptive strategies $(P=.009$, $95 \% \mathrm{CI}=0.03-0.22)$. Benefits were sustained at 12 months for most outcomes.

CONCLUSION: A multicomponent intervention targeting modifiable environmental and behavioral factors results in life quality improvements in community-dwelling older people who had functional difficulties, with most benefits retained over a year. 
Functional disability is a major adverse outcome of age-related chronic and debilitating conditions that are common in older people, including cardiovascular disease, osteoarthritis, and stroke. ${ }^{1}$ Difficulties performing instrumental activities of daily living (IADLs) or activities of daily living (ADLs) such as bathing or ambulating at home represent sentinel events that may trigger the need for personal assistance or relocation to a family member's home or residential facility. ${ }^{2,3}$ Moreover, it is associated with a diminished quality of life, poor self-efficacy, and high healthcare costs and service utilization. ${ }^{4}$ Additionally, functional difficulties are associated with fear of falling, a geriatric syndrome with pernicious psychological and physical consequences including social isolation, anxiety, depression, functional decline, and falls. $^{5-7}$

Health providers and policy makers widely recognize the importance of ameliorating functional difficulties associated with chronic illness. This is reflected in the requirements of the Center for Medicare and Medicaid Services and third-party reimbursement mechanisms that functional difficulties serve as an eligibility criteria for home care services. ${ }^{8,9}$ Nevertheless, older people with functional disabilities do not typically receive home care to address performance difficulties unless an acute medical episode or hospitalization triggers a referral for therapies. Furthermore, under 
current guidelines, services are brief and designed to address acute rather than longterm consequences of chronic conditions. This is problematic in that recent research shows that older people perform self-care at close to their maximum capacity such that even small declines in health or changes in their home environments may contribute to the disablement process. ${ }^{10}$

At the same time, it remains unclear as to the most effective approach to minimize functional difficulties. Previously tested interventions to address functional decline have focused on condition-specific clinical populations, ${ }^{11,12}$ have been implemented at hospital discharge ${ }^{13}$ or as part of formal home care services, ${ }^{14}$ or have targeted underlying impairments, ${ }^{15}$ with inconsistent outcomes reported. ${ }^{16-19}$ Studies of nurse home visitation programs, implemented primarily in Europe, have also yielded inconclusive findings. ${ }^{17-19}$ These trials suggest that there is no single approach that effectively addresses functional problems. The most successful interventions are multicomponent and target areas that individuals self-identify as problematic. Thus, it remains an important public health priority to identify effective interventions to manage ongoing and long-term functional consequences of chronic disease and improve life quality of older community members. ${ }^{4,19}$

A promising new direction involves targeting modifiable behavioral and environmental contributors to functional decline. Recent research shows that environmental features such as an inaccessible or unsafe home may exacerbate functional difficulties and lead to further decline. ${ }^{4,16} \mathrm{~A}$ few intervention studies 
involving home modifications show reductions in risk for falls ${ }^{20}$ and maintenance of functioning for persons with frailty and dementia. ${ }^{21,22}$

A two-group, randomized, controlled trial was conducted to evaluate a multicomponent home-based intervention that was designed to reduce difficulties in performing everyday tasks in community-dwelling people aged 70 and older by modifying behavioral and environmental contributors to functional decline. It was hypothesized that at 6 months, intervention participants would report less difficulty with daily activities, more confidence in managing self-care, less fear of falling, and greater use of adaptive strategies than a no-treatment control group. It was also hypothesized that the number of observed home hazards would be reduced. Also examined was whether treatment benefits achieved at 6 months were sustained at 12 months.

\section{METHODS}

Study Sample and Procedures

Participants were recruited between 2000 and 2003 from an area agency on aging, media announcements, and posters at senior housing and community settings. Study procedures were explained to interested persons whom the research team contacted or who called the research office, and a brief telephone screen was administered to determine eligibility. All participants were aged 70 and older, cognitively intact (MiniMental State Examination (MMSE) score 423 on a scale ranging from 0 to 30 ), ${ }^{23}$ and English speaking; were not receiving home care; and reported the need for help or 
difficulties with two IADLs or one or more ADLs. ${ }^{24}$ These criteria were designed to enroll older people who experienced some difficulty with everyday activities but who were not totally dependent or homebound or receiving services to address functional problems.

Of the 636 persons telephone screened, 423 were potentially eligible, of whom 331 (78.3\%) were willing to participate. There were no large or statistically significant differences in self-reported functional difficulties between the 331 eligible and willing participants and the 92 persons eligible but unwilling to participate. Of the 331 persons who received the baseline home interview, 4\% (n 5 12) were ineligible based on MMSE scores and were excluded, resulting in a sample of 319 . Written informed consent was obtained at baseline using an approved institutional review board form. The project codirectors (LW, SS) randomized participants, and trained interviewers who were masked to group assignment and study hypotheses and who had no role in the intervention interviewed them at 6 and 12 months.

\section{Randomization}

Study participants were stratified by race (white, nonwhite) and living arrangement (alone, with others) and randomized within each of four strata using random permuted blocks to control for possible changes in subject mix over time. The blocking number, developed by the project statistician $(\mathrm{WWH})$, remained unknown to others. Randomization lists and four sets of randomization were prepared using double, opaque envelopes. The project director randomized each participant within 48 
hours from completion of the baseline interview.

\section{Intervention Group}

Research on behavioral and environmental factors that place demands on individuals that exceed their capabilities and thus contribute to disability guided the intervention. The Life Span Theory of Control as applied to the disablement process was also used. This framework proposes that the progression from pathology to disability increases the threat to personal control, which in turn may result in negative health and affective consequences, particularly for older people. ${ }^{25}$ Individuals become motivated to use control-oriented strategies to maintain or enhance behavior-event contingencies in everyday life domains. Thus, the intervention goal was to compensate for declining abilities by training in the use of control-enhancing strategies including cognitive (problem-solving, reframing), behavioral (pace self, sit instead of stand to perform tasks), and environmental (grab bars) modifications.

The 6-month intervention consisted of five occupational therapy contacts (four 90minute visits and one 20-minute telephone contact) and one physical therapy visit (90 minutes). Occupational therapists (OTs) initially met with participants and conducted a semistructured clinical interview to identify and prioritize problem areas. For each targeted area, an OT observed the participant's performance for safety, efficiency, and difficulty and presence of environmental barriers. In subsequent sessions, the OT engaged the participant in problem solving to identify behavioral and environmental contributors to performance difficulties. Specific strategies were 
derived and equipment options provided. In the fourth session, the physical therapist (PT) provided balance and muscle strengthening and fall-recovery techniques. In the fifth session (telephone), the OT reinforced strategy use, and in the sixth session, the OT reviewed problem solving, refined strategy use, and provided education and resources to address future needs for environmental adjustments. Before the sixth contact, the area agency on aging ordered and installed home modifications (grab bars, rails, raised toilet seats), which were paid for through grant funds.

Over the following 6 months, OTs conducted three telephone calls to reinforce the use of intervention-derived strategies and generalize these strategies to new problem areas. A final home visit was conducted to obtain closure.

Because of considerable variability in home environments and functional difficulties, specific control-oriented strategies were individualized to the needs of participants, although the intervention was standardized in that each participant received four treatment components (education and problem solving; home modification; energy conserving techniques; and balance, muscle strengthening, and fall-recovery techniques) for specific targeted functional areas.

Although the intervention program was based on traditional occupational and physical therapy techniques, ${ }^{26-28}$ its approach differed from typical home care. First, the intervention focused exclusively on the areas participants themselves reported as problematic. In traditional home care, problem areas addressed by health professionals may not reflect client priorities. Second, interventionists served as consultants, helped participants solve problems, and offered strategy choices, 
whereas home care is more directive and prescriptive. Third, the intervention involved coordination between OTs and PTs to achieve an integrated approach, which is not always possible in home care.

Participants who were assigned to the no-treatment control group did not receive any intervention contact. At the conclusion of the 12-month follow-up interview, control participants were provided educational materials on home safety and safe performance techniques.

Treatment Implementation

Interventionists were licensed therapists with 1 or more years of home care experience. Interventionists received 35 hours of training that involved readings, didactic instruction, practice, role-play, and calibration in use of the semi-structured clinical interview. Treatment implementation was monitored and maintained in supervision meetings held every other week in which cases were systematically presented. Interventionists also submitted taped treatment sessions for review and feedback by the investigators. Additionally, documentation was completed for each contact and reviewed to ensure compliance with treatment delivery.

\section{Outcomes}

Study participants were evaluated using standardized measures. Primary outcomes included self-report measures of functional difficulties, self-efficacy, and fear of falling, and secondary outcomes included adaptive strategy use and observed home hazards. 
With regard to the primary outcomes, three areas of physical function were assessed: ADLs, mobility/transferring, and IADLs. ${ }^{29}$ For each area, participants rated their perceived difficulty in the previous month from $1=$ no difficulty to $5=$ unable to do because of health problems. High scores indicated greater difficulty. An ADL index was computed as mean difficulty across six items (dressing above waist, dressing below waist, grooming, bathing/showering, toileting, and feeding; Cronbach $a=$ 0.67). A mobility/ transfer index was computed as mean difficulty across six items (getting in/out of car, walking indoors, walking one block, climbing one flight of stairs, moving in/out of chair, and moving in/out of bed; Cronbach $a=0.68)$. The IADL index was calculated as the mean across six items (light housework, shopping, preparing meals, managing money, telephone use, and taking medications; Cronbach $a=0.58)^{29,30}$

Fear of falling was measured using Tinetti et al.'s Falls Efficacy Scale plus three items from Powell et al.'s Activities-specific Balance Confidence Scale (confident walking up/down stairs, bending/picking up slipper from floor, and getting into/out of car without falling). ${ }^{5,31}$ For each item, participants rated their confidence in performing activities without falling along a 10-point Likert scale. The fear of falling index represented the mean response across 13 items. High scores indicated less fear of falling (Cronbach $a=0.93$ ).

Self-efficacy refers to an assessment of one's ability to perform a particular activity and achieve a desired outcome. ${ }^{32}$ For 17 functional activities (IADLs, ADLs, and 
mobility), participants rated their confidence in managing difficulties from $1=$ not at all confident to $5=$ very confident. The functional self-efficacy index represented average perceived confidence across items. High scores indicated greater confidence (Cronbach $a=0.90)$.

With regard to secondary endpoints, the presence of 106 potential tripping and falling hazards (torn carpets, glare, lack of grab bars) was observed using a reliable instrument with strong interrater reliability. ${ }^{33}$ The home hazard index represented the total number of potentially unsafe conditions (Cronbach $a=0.71$ ).

An eight-item investigator-developed measure assessed adaptive behavioral, cognitive, and environmental strategy use (unpublished data). Participants rated the extent to which items (I try to make changes to my home to help me stay independent) were true, from $1=$ not at all true to $4=$ very much true. A controloriented strategy index was derived by averaging responses across the eight items. Higher mean scores indicated greater strategy use (Cronbach $a=0.69$ ).

\section{Statistical Analysis}

The sample size was calculated based on the following assumptions: $40 \%$ attrition rate, hypotheses tested at the .05 level (1-tailed), and $90 \%$ power to detect medium effects ( 0.5 standard deviation) in the primary endpoints (functional difficulties, selfefficacy, and fear of falling). ${ }^{34}$ An $a=0.05 / 350.0167$ level, one-sided test, was used. Because the planned analyses were analyses of covariance (ANC-OVAs), the power of a two-sample t test was calculated. A total of 190 subjects ( 95 per group) 
were required to have $90 \%$ power to detect a medium effect ( 0.5 standard deviation). Given the initial expectation of a substantial attrition rate, it was planned to randomize 319 subjects.

Descriptive data included sociodemographic characteristics (age, sex, race, living arrangement), health conditions, availability of social supports, and depressive symptoms (measured using the Radloff Center for Epidemiological Studies Depression Scale). ${ }^{35}$ Chi-square and Wilcoxon rank-sum tests were used to compare experimental and control participants on baseline characteristics. Means, standard deviations, and ranges for each outcome measure were computed. Main treatment effects at 6 months were examined using ANCOVA. The outcome measure was the 6-month score with design variables (race and living arrangements) used as covariates for all outcome analyses. To increase precision of treatment comparisons, additional covariates were chosen a priori based on previous research that showed a relationship with outcomes. For functional difficulty analyses (ADL, IADL, and mobility/ transferring), economic well-being, social support, and depression were added as covariates. Economic well-being serves as a proxy for social class, with previous research showing that greater affluence is associated with higher physical functioning. Whereas research shows that social support buffers functional decline, depression is a known risk factor. For self-efficacy and fear of falling, functional difficulty and depression are known predictors and were included as covariates. For the home hazard analysis, number of recent falls and near falls, perceived ability to manage fall risk, and health conditions were selected as covariates because of their documented 
associations with environmental factors. Although the distribution of residuals was somewhat skewed for ADL difficulty, neither a log nor square root transformation improved the distribution, and the nontransformed distribution is reported.

To evaluate whether treatment effects remained at 12 months, intervention and control groups were compared on adjusted mean differences from baseline to 12 months for each outcome variable using the above procedures.

SPSS version 13.0 was used, with significance level set at .05 (SPSS, Inc., Chicago, IL). All analyses were two-sided. Analyses followed intention to treat such that all subjects providing data were included in analyses regardless of study participation level.

\section{RESULTS}

Participants were primarily female $(81.8 \%)$ and lived alone $(61.8 \%)$. Half $(52.7 \%)$ identified as white, and $45.5 \%$ were African American (Table 1). Approximately onethird of the sample had less than a high school education, about one-third had a high school education, and slightly more than one-third had more than a high school education. On average, study participants were aged 79, reported some to a lot of difficulty ambulating (mean \pm standard deviation $2.5 \pm 0.8$ ), carrying out self-care $(1.8 \pm 0.6)$, and with IADLs $(2.1 \pm 0.6)$. Also, on average, participants reported seven health conditions, with $84 \%$ reporting arthritis, $71 \%$ reporting hypertension, $43 \%$ reporting cataracts or macular degeneration, 39\% reporting cardiovascular problems, and $23 \%$ reporting diabetes mellitus. Furthermore, $69.6 \%$ of participants 
rated their health as fair to poor, and $51 \%$ indicated that their health was not as good as 1 year before.

Of the 319 participants (159 control, 160 experimental), 300 (94\%) were available at 6 months and $285(89 \%)$ at 12 months. Discontinuation of 34 participants by 12 months was accounted for by 14 who died, eight lost to follow-up, five who entered a nursing home, four who were dissatisfied, two who were unable to complete the batteries due to poor health, and one who was hospitalized (Figure 1).

A comparison of active participants with the 34 who discontinued by 12 months revealed that active participants had lower MMSE scores (mean 26.8) and more health conditions (mean 7.1) than those discontinuing (mean 27.8; $\mathrm{P}=.003 ;$ mean $6.0 ; P=.01$, respectively).

At 6 months, intervention participants $(n=154)$ had less difficulty with IADLs and ADLs than controls $(n=146)$ (Table 2$)$, with largest benefits occurring in bathing ( $P$ $=.02,95 \%$ confidence interval $(C I)=-.52$ to -.06$)$ and toileting $(P=.049,95 \% C I=$ -.35-0.00). Although mobility/transfer difficulty scores were lower for intervention participants than for controls, the difference was not statistically significant. Also, intervention participants reported less fear of falling, greater confidence in managing daily functional activities, and greater use of control-oriented strategies than controls. Additionally, fewer home hazards were observed for this group than for controls (Table 2), particularly in bathrooms. Furthermore, a greater proportion of intervention participants showed improvements (Figure 2) in 11 of 18 specific activities than of controls, with statistical significance obtained for bathing $(P=.04)$, grooming $(P=$ 
$.04)$, and preparing meals $(P=.02)$.

For the 285 participants available at 12 months, the magnitude of the 12 -month treatment effects was similar to the 6-month effects for three of five of the primary outcomes (ADL and IADL functional difficulty, fear of falling) and for the two secondary outcomes (home hazards and control-oriented strategy use), whereas the magnitude was half that at 6-month results for function-related self-efficacy (Table 2).

Costs associated with the 6-month intervention program were minimal. Average costs for equipment and home modifications were $\$ 439$ (device, delivery, and installation) per participant. Therapy costs per participant were $\$ 783$ using the Medicare reimbursement schedule for home care services ( $\$ 25$ per 15 -minute therapeutic unit). Thus, the mean 6-month intervention cost was $\$ 1,222$ per experimental participant.

\section{DISCUSSION}

This study targeted a service-neglected group, community-living older people with functional difficulties who were not receiving home care services. Functional difficulties and fear of falling represent major threats to the quality of life of older adults and should be addressed concomitantly with disease treatment. Although the prevalence of functional disability in older people is declining, the absolute size of this group is increasing and is expected to exceed 12 million by $2030 .{ }^{36,37}$ Fear of falling, which increases with age, represents a pernicious consequence of frailty that further 
contributes to activity limitations, functional decline, and risk for falls. ${ }^{38}$

Although the majority of older people age at home, they increasingly confront functional difficulties that place them at risk of frailty and relocation. This randomized, controlled trial provides evidence that a low-cost $(\$ 1,222$ per patient $)$ occupational/physical therapy home intervention that addresses modifiable environmental and behavioral risk factors is effective in reducing perceived functional difficulties and enhancing self-efficacy and fall-related concerns in older people who may be transitioning to frailty. Intervention participants had greater improvements in tasks that are critical for independent living, such as bathing and toileting with less difficulty. Furthermore, intervention participants reported greater self-efficacy in managing day-today self-care. ${ }^{39,40}$ Also, the intervention enhanced engagement in control-oriented strategies, which previous research has shown to be associated with beneficial health outcomes. ${ }^{41}$ Equally important was a reduction in fall risk factors, including home hazards, ${ }^{42}$ and difficulty ambulating in the home. Finally, intervention participants reported less fear of falling than controls, who showed increased fear at 6 and 12 months. Fear of falling, a recognized psychological syndrome, is a strong risk factor for falling and functional decline. Only one other intervention, A Matter of Balance, ${ }^{43}$ which used a community-based group format, showed reductions in fear of falling. The current intervention could provide an alternative approach for those unable or unwilling to attend group sessions in the community.

It was also found that the magnitude of 12-month effects was similar to those at 6 
months for five of the seven outcomes with minimal therapy contact. Although effect sizes for all treatment outcomes were small to medium (ranging from 0.19 to 0.26 ), the results compare favorably with other in-home intervention studies on functional decline $^{17}$ and suggest that targeting modifiable factors such as the environment and introducing behavioral and cognitive strategies including techniques for fall recovery are worthy of consideration.

It is unclear whether one intervention component was more effective than others, although it is likely that it was the multicomponent approach and targeting of functional tasks that older people themselves perceived as problematic that made the difference. ${ }^{44}$ Similar to other geriatric syndromes, a multicomponent approach may more effectively address functional difficulties.

One potential limitation of this study was the use of a no-treatment control group, which leaves open the possibility that attention from health professionals may account for the beneficial effects, although it is unlikely that reported improvements in daily functioning, particularly in bathing and toileting, and observed home hazard reductions were a consequence of attention alone. Moreover, intervention participants reported greater use of control-oriented strategies, whereas control group participants reported little change in use of these strategies at 6 or 12 months. These strategies reflect the techniques that were actively introduced in the intervention (e.g., pacing oneself, home modification use) and thus support the argument that attention or the halo effect alone cannot explain treatment benefits. A related concern is that the primary study outcomes were subjective self-reports of difficulty with daily life tasks 
and that performance-based measures were not included. Other studies have shown that self-rated function is predictive of negative health consequences including mortality. ${ }^{45}$ Moreover, functional difficulty represents an important dimension of disability and is used to determine competency and service needs. ${ }^{46}$ Still, future research needs to consider objective and subjective indicators, given the multidimensionality of physical function. ${ }^{47}$ Also, the combination of the lack of an attention control group and objective performance measures suggest that future research to confirm these findings should be pursued.

Another limitation concerns the extent to which findings can be generalized to a wider population of vulnerable older adults. Given that study participation was voluntary, it may be that this sample was more motivated to learn new strategies than nonvolunteers. Control group participants also reported improvement in select functional areas (Figure 2), although the difference from baseline was not statistically significant, and on average there were slight improvements in ADLs and mobility and none in IADLs (Table 2). One explanation may be that difficulties fluctuate over time. Another explanation may be that the baseline interview, and in particular questions about adaptive strategies, may inadvertently lead to behavioral and cognitive adjustments that result in functional improvements.

Several implications for healthcare delivery may be derived from these findings. A group of older people who were not receiving occupational and physical therapy services but who nevertheless had difficulties with everyday activities was targeted. Typically, older people are referred for reimbursable therapy services after a 
hospitalization or sentinel event, but these findings suggest that occupational and physical therapy professional services should have a greater and more active role in chronic disease management. Because this program improved functional performance in everyday activities of living, the services are reimbursable under Medicare guidelines. Thus, older patients who report functional difficulties or fear of falling may benefit from a referral for occupational and physical therapy services delivered by those trained in this program. Because home modifications are typically not reimbursable and involve out-of-pocket expenditures, the findings support a change in this policy. The intervention does not reflect new technologies or treatments; it uses existing treatment modalities more effectively and in a coordinated, systematic, integrated fashion to address daily concerns of older people. Results show services to be feasible, well received, effective across a range of outcomes, and probably cost effective. The study also shows that older people can learn simple behavioral and environmental strategies to address everyday difficulties.

In summation, functional difficulties imposed by chronic health problems are a primary threat to quality of life and warrant attention from the medical community. Study results suggest that modifying environmental and behavioral factors can ameliorate functional difficulties and concomitant concerns such as fear of falling, poor self-efficacy, and home hazards. Future research needs to determine cost effectiveness, include objective functional performance measures, determine whether some older people benefit more than others, identify the mechanisms by which the intervention achieves its outcomes, and explore the unique contribution of treatment 
components.

\section{ACKNOWLEDGMENTS}

The research reported in this paper was supported by funds from National Institute on Aging Grant R01 AG13687. We acknowledge the important contributions of Susan Klein, MA, Director of Housing, Philadelphia Corporation for Aging (area agency on aging), who coordinated equipment orders and installation and assisted with recruitment; interventionists Nancy Prickett, PT/L, Gina Colier, OTR/L, Mary Ferraro, PhD, OTR/L, Pamela Kearney, MA, OTR/L, Geri Shaw, OTR/L, Tracey Vause-Earland, MA, OTR/L, Lois Rosage, OTR/L, and Susan Santalucci, OTR/L. We also gratefully thank the interviewing staff and study participants for their time and responses.

Financial Disclosures: None.

Author Contributions: L. N. Gitlin, principal investigator: developed study concept and design, and oversaw scientific integrity and interpretation of data, primary responsibility for preparation of manuscript. L. Winter, coproject director: assisted in acquisition of subjects, data analysis, and manuscript preparation. M. Dennis: conducted data analysis and constructed manuscript tables. M. Corcoran, coinvestigator: helped design intervention, oversaw treatment implementation, reviewed manuscript for accuracy. S. Schinfeld, coproject director: assisted with subject recruitment, supervised interviewers, and reviewed manuscript for accuracy. W. Hauck, statistician: oversaw design development, statistical analyses, and integrity of data interpretation. 
Sponsor's Role: None.

\section{REFERENCES}

1. Fried LP, Guralnik JM. Disability in older adults. Evidence regarding significance etiology, and risk. J Am Geriatr Soc 1997;45:92-100.

2. Naik AD, Concato J, Gill TM. Bathing disability in community-living older persons: Common, consequential and complex. J Am Geriatr Soc 2004; $52: 1805-1810$

3. Gill TM, Kurland B. The burden and patterns of disability in activities of daily living among community-living older persons. J Gerontol A Biol Sci Med Sci 2003;58A:M70-M75.

4. Spillman BC. Changes in elderly disability rates and the implications for health care utilization and cost. Milbank Q 2004;82:157-194.

5. Tinetti ME, Richman D, Powell L. Falls efficacy as a measure of fear of falling. J Gerontol 1990;45:P239-P243.

6. Arfken $\mathrm{CL}$, Lach HW, Birge SJ et al. The prevalence and correlates of fear of falling in elderly persons living in the community. Am J Public Health $1994 ; 84: 565-570$.

7. Bruce DG, Devine A, Prince RL. Recreational physical activity levels in healthy older women. The importance of fear of falling. J Am Geriatr Soc 2002;50: 8489.

8. Vladeck BC, Miller NA. The Medicare home health initiative. Health Care Financ 
Rev $1994 ; 16: 7-16$.

9. The Commonwealth Fund. Facts on Medicare's Home Health Benefit and Recent Policy Changes June 1998 [on-line]. Available at www.cmwf.org/publications/publications_show.htm?doc_id=221594 Accessed July 14, 2004.

10. Hortobagyi T, Mizelle C, Beam S et al. Old adults perform activities of daily living near their maximal capabilities. J Gerontol A Biol Sci Med Sci 2003;58A:M453-M460.

11. Rich MW, Beckham V, Wittenberg $C$ et al. A multi-disciplinary intervention to prevent the readmission of elderly patients with congestive heart failure. $\mathrm{N}$ Engl J Med 1995;333:1190-1195.

12. Tinetti ME, Baker DI, Gottschalk M et al. Home-based multicomponent rehabilitation program for older persons after hip fracture: A randomized trial. Arch Phys Med Rehabil 1999;80:916-922.

13. Kravitz RL, Reuben DB, Davis JW et al. Geriatric home assessment after hospital discharge. J Am Geriatr Soc 1994;42:1229-1234.

14. Tinetti ME, Baker D, Gallo WT et al. Evaluation of restorative care vs usual care for older adults receiving an acute episode of home care. JAMA $2002 ; 287: 2098-2105$.

15. Gill TM, Baker DI, Gottschalk M et al. A program to prevent functional decline in physically frail, elderly persons who live at home. N Engl J Med $2002 ; 347: 1068-1074$ 
16. van Haastregt JC, Diederiks JP, van Rossum E et al. Effects of preventive home visits to elderly people living in the community: A systematic review. BMJ $2000 ; 320: 754-758$.

17. Stuck $A E$, Egger $M$, Hammer $A$ et al. Home visits to prevent nursing home admission and functional decline in elderly people: Systematic review and metaregression analysis. JAMA 2002;287:1022-1028.

18. Elkan $R$, Kendrick D, Dewey $M$ et al. Effectiveness of home based support for older people: Systematic review and meta-analysis. BMJ 2001;323:719724.

19. Ferrucci L, Guralnik JM, Studenski S et al. The Interventions on Frailty Working Group. Designing randomized, controlled trials aimed at preventing or delaying functional decline and disability in frail, older persons: A consensus report. J Am Geriatr Soc 2004;52:625-634.

20. Cumming RG, Thomas M, Szonyi G et al. Home visits by an occupational therapist for assessment and modification of environmental hazards: A randomized trial of falls prevention. J Am Geriatr Soc 1999;47:1397-1402.

21. Mann WC, Ottenbacher KJ, Fraas L et al. Effectiveness of assistive technology and environmental interventions in maintaining independence and reducing home care costs for the frail elderly. A randomized controlled trial. Arch Fam Med 1999;8:210-217.

22. Gitlin LN, Winter L, Corcoran MA et al. Effects of the Home Environmental Skill-building Program on the caregiver-care recipient dyad: Six month 
outcomes from the Philadelphia REACH initiative. Gerontologist 2003;43:532546.

23. Folstein MF, Folstein SE, McHugh PR. 'Mini-mental state'. A practical method for grading the cognitive state of patients for the clinician. J Psychiatr Res $1975 ; 12: 189-198$.

24. Morris JN, Sherwood S, Mor V. An assessment tool for use in identifying functionally vulnerable persons in the community. Gerontologist $1984 ; 24: 373-$ 379.

25. Schulz R, Heckhausen J, O'Brien AT. Control and the disablement process in the elderly. J Soc Behav Pers 1994;9:139-152.

26. Corcoran MA, Gitlin LG. The role of the physical environment in occupational performance. In: Christiansen C, Baum C, eds. Occupational Therapy. Overcoming Human Performance Deficits, 2nd Ed. Thorofare, NJ: Slack Inc, 1997, pp 336-361.

27. Hinojosa J, Kramer P, Royeen CR et al. Core concept of occupation. In: Kramer P, Hinojosa J, Royeen CR, eds. Perspective in Human Occupation: Participation in Life. Philadelphia, PA: Lippincott, Williams \& Wilkins, 2003, pp $1-17$.

28. Shumway-Cook A, Woollacott MH. Motor Control: Theory and Practical Applications. Baltimore, MD: Williams \& Wilkins, 1995.

29. Ettinger WH Jr, Burns R, Messier SP et al. A randomized trial comparing aerobic exercise and resistance exercise with a health education program in 
older adults with knee osteoarthritis. The Fitness Arthritis and Seniors Trial (FAST). JAMA 1997;227:25-31.

30. Lawton MP, Brody E. Assessment of older people: Self-maintaining and instrumental activities of daily living. Gerontologist 1969;9:179-186.

31. Powell LE, Myers AM. The Activities-specific Balance Confidence (ABC)

Scale. J Gerontol A Biol Sci Med Sci 1995;50A:M28-M34.

32. Bandura A. Self-Efficacy. The Exercise of Control. New York: W.H.

Freeman, 1997.

33. Gitlin LN, Schinfeld S, Winter L et al. Evaluating home environments of persons with dementia: Interrater reliability and validity of the Home Environmental Assessment Protocol (HEAP). Disabil Rehabil 2002;24:59-71.

34. Cohen J. Statistical Power Analyses for the Behavioral Sciences, Revised Ed. Hillsdale, NJ: Erlbaum, 1987.

35. Radloff LS. The CES-D Scale: A self-report depression scale for research in the general population. Appl Psychol Meas 1997;1:385-401.

36. Federal Interagency Forum on Aging Related Statistics. Older Americans 2000: Key indicators of well-being. Available at www.agingstats.gov/chartbook2000/OlderAmericans2000.pdf Accessed July 14, 2004.

37. Manton KG, Corder L, Stallard E. Chronic disability trends in elderly United States populations: 1982-94. Proc Natl Acad Sci U S A 1997;94:2593-2598.

38. Kressig RW, Wolf SL, Sattin RW etal. Associations of demographic, 
functional, and behavioral characteristics with activity-related fear of falling among older adults transitioning to frailty. J Am Geriatr Soc 2001;49:14561462.

39. Verbrugge LM, Rennert C, Madans JH. The great efficacy of personal and equipment assistance in reducing disability. Am J Public Health 1997;87:384392.

40. Lorig KR, Sobel DS, Ritter PL et al. Effect of a self-management program on patients with chronic disease. Eff Clin Pract 2001;4:256-262.

41. Wrosch C, Schulz R, Heckhausen J. Health stresses and depressive symptomatology in the elderly: The importance of health engagement control strategies. Health Psychol 2002;21:340-348.

42. Marshall SW, Runyan CW, Jingzhen $Y$ et al. Prevalence of selected risk and protective factors for falls in the home. Am J Prev Med 2005;28:95-101.

43. Tennstedt S, Howland J, Lachman M et al. A randomized, controlled trial of a group intervention to reduce fear of falling and associated activity restriction in older adults. J Gerontol B Psych Sci Soc Sci 1998;53B:P384-P392.

44. Bradley EH, Bogardus ST Jr, Tinetti ME et al. Goal-setting in clinical medicine. Soc Sci Med 1999;49:267-278.

45. Greiner PA, Snowdon DA, Greiner LH. The relationship of self-rated health to concurrent functional ability, functional decline, and mortality: Findings from The Nun Study. J Gerontol B Psychol Sci Soc Sci 1996;51B:S234-S241. 46. Gill TM, Robinson JT, Tinetti ME. Difficulty and dependence: Two 
components of the disability continuum among community-living older persons.

Ann Intern Med 1998;128:96-101.

47. Gitlin LN. Physical Function in Older Adults: A Comprehensive Guide to Its Meaning and Measurement. Austin, TX: ProEd, 2005.

TABLES \& FI GURES

Table 1. Characteristics of Baseline Sample ( N 5 319)

\begin{tabular}{|c|c|c|c|c|}
\hline Characteristic & $\begin{array}{c}\text { Control } \\
(n=159)\end{array}$ & $\begin{array}{l}\text { Experimental } \\
(\mathrm{n}=160)\end{array}$ & $\begin{array}{c}\text { Total } \\
(\mathrm{N}=319)\end{array}$ & P-value \\
\hline Age, mean \pm SD & 78.55 .7 & 79.56 .1 & 79.05 .9 & $\begin{array}{l}.16 \\
.99\end{array}$ \\
\hline Race, \% & & & & \\
\hline White & 52.2 & 53.1 & 52.7 & \\
\hline African American & 45.9 & 45.0 & 45.5 & \\
\hline Other & 1.9 & 1.9 & 1.8 & \\
\hline Sex, \% & & & & .75 \\
\hline Male & 18.9 & 17.5 & 18.2 & \\
\hline Female & 81.1 & 82.5 & 81.8 & \\
\hline Living arrangement, \% & & & & .46 \\
\hline Alone & 59.7 & 63.8 & 61.8 & \\
\hline With others & 40.3 & 36.2 & 38.2 & \\
\hline Education, \% & & & & .92 \\
\hline$<$ High school & 30.2 & 31.9 & 31.0 & \\
\hline High school & 32.1 & 32.5 & 32.3 & \\
\hline$>$ High school & 37.7 & 35.6 & 36.7 & \\
\hline $\begin{array}{l}\text { Mini-Mental State Examination } \\
\text { score, mean } \pm \text { SD } \\
\text { Number of health conditions. }\end{array}$ & 27.01 .82 & 26.81 .8 & 26.91 .8 & .35 \\
\hline mean \pm SD & 7.12 .8 & 6.72 .7 & 6.92 .7 & .30 \\
\hline
\end{tabular}

SD 5 standard deviation. 
Figure 1. Flow chart of recruitment and study participation.

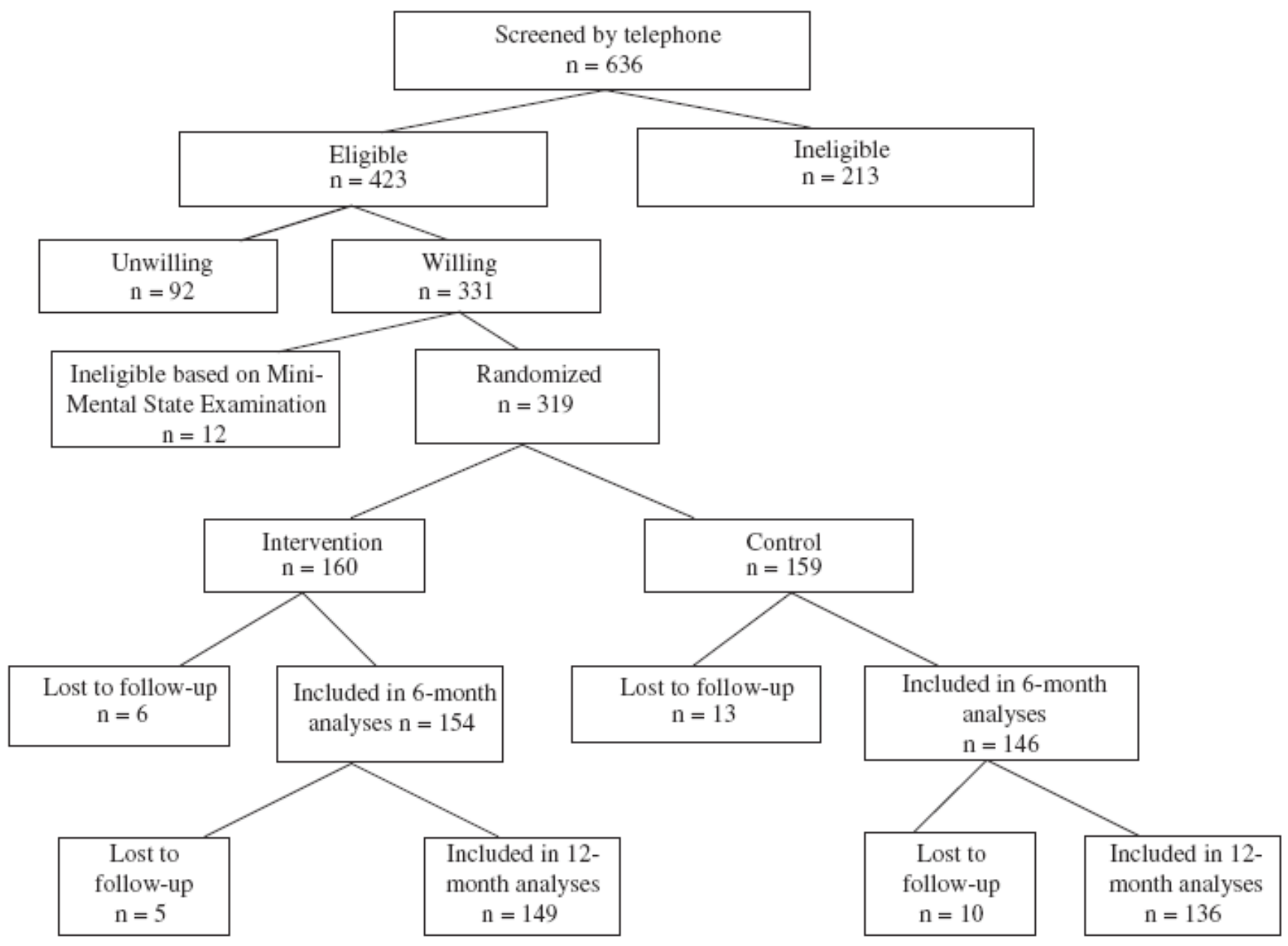

Author's final version prior to publication in Journal of the American Geriatrics Society 54(5):809-816, May 2006. Copyright (C) 2006. The published version is available at http://dx.doi.org/10.1111/j.1532-5415.2006.00703.x 


\section{Table 2. Outcomes for Experimental and Control Group Participants at 6 and 12 Months}

\begin{tabular}{|c|c|c|c|c|c|c|c|c|}
\hline \multirow[b]{2}{*}{ Dependent Variable } & & \multirow[b]{2}{*}{ Control } & \multirow[b]{2}{*}{ Range } & \multirow{2}{*}{$\begin{array}{l}6 \text { Months } \\
(\mathrm{N}=300) \\
\text { Difference of } \\
\text { Adjusted } \\
\text { Means at } \\
6 \text { Months* }\end{array}$} & \multirow{2}{*}{$\begin{array}{c} \\
P \text { - } \\
\text { value }\end{array}$} & \multicolumn{2}{|c|}{$\begin{array}{l}12 \text { Months } \\
(N=285)\end{array}$} & \multirow[b]{2}{*}{$\begin{array}{c}95 \% \\
\text { Confidence } \\
\text { Interval }\end{array}$} \\
\hline & $\begin{array}{l}\text { Experimental } \\
\text { Mean } \pm \text { Stand }\end{array}$ & & & & & $\begin{array}{l}\text { Difference } \\
\text { of Adjusted } \\
\text { Means at } \\
12 \text { Months* }\end{array}$ & $\begin{array}{c}P- \\
\text { value }\end{array}$ & \\
\hline Baseline & $1.82 \pm 0.57$ & $1.76 \pm 0.61$ & & & & & & \\
\hline 6 months & $1.58 \pm 0.54$ & $1.66 \pm 0.63$ & & & & & & \\
\hline Mobility & & & & $-0.11^{\dagger}$ & .15 & $-0.14^{\dagger}$ & .07 & $-0.29-0.01$ \\
\hline Baseline & $2.55 \pm 0.73$ & $2.49 \pm 0.78$ & & & & & & \\
\hline 6 months & $2.35 \pm 0.72$ & $2.41 \pm 0.80$ & & & & & & \\
\hline \multicolumn{9}{|l|}{ Self-efficacy } \\
\hline Fear of falling & & & $1.00-10.00$ & $0.61^{\ddagger \leftarrow}$ & $.001^{\top}$ & $0.56^{\ddagger \leftarrow}$ & $.008^{\mp}$ & $0.15-0.97$ \\
\hline Baseline & $7.21 \pm 2.0$ & $7.58 \pm 1.85$ & & & & & & \\
\hline 6 months & $7.79 \pm 1.83$ & $7.45 \pm 2.13$ & & & & & & \\
\hline Overall functional efficacy & & & $1.00-5.00$ & $0.15^{t \leftarrow}$ & $.02^{\uparrow}$ & $0.09^{t \leftarrow}$ & .23 & $-0.06-0.23$ \\
\hline Baseline & $3.92 \pm 0.72$ & $4.03 \pm 0.67$ & & & & & & \\
\hline 6 months & $4.23 \pm 0.63$ & $4.16 \pm 0.70$ & & & & & & \\
\hline \multicolumn{9}{|l|}{ Environmental } \\
\hline Home hazards & & & $0.00-106.00$ & $-1.53^{\S}$ & $.05^{\uparrow}$ & $-1.38^{\S}$ & .13 & $-3.17-0.41$ \\
\hline Baseline & $11.37 \pm 8.25$ & $10.86 \pm 8.16$ & & & & & & \\
\hline 6 months & $8.55 \pm 7.25$ & $9.86 \pm 7.88$ & & & & & & \\
\hline Control-oriented strategies & & & $1.00-4.00$ & $0.13^{\| \leftarrow}$ & $.009^{\top}$ & $0.14^{\| \leftarrow}$ & $.01^{\leftarrow}$ & $0.03-0.24$ \\
\hline
\end{tabular}

Author's final version prior to publication in Journal of the American Geriatrics Society 54(5):809-816, May 2006. Copyright (C) 2006. The published version is available at http://dx.doi.org/10.1111/j.1532-5415.2006.00703.x 
* Adjusted for baseline value.

${ }^{\dagger}$ Adjusted for race, living arrangement, economic well-being, social support, and depressive symptoms.

${ }^{\ddagger}$ Adjusted for race, living arrangement, functional difficulty, and depressive symptoms.

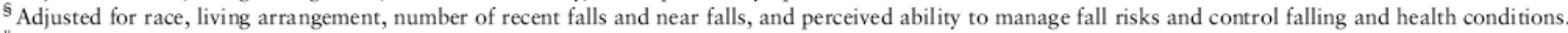

"Adjusted for race and living arrangement.

`Statistically significant.

Figure 2. Comparison of percentage improvement in functional activities between experimental and control group participants at 6 months $(\mathrm{N}=300)$.

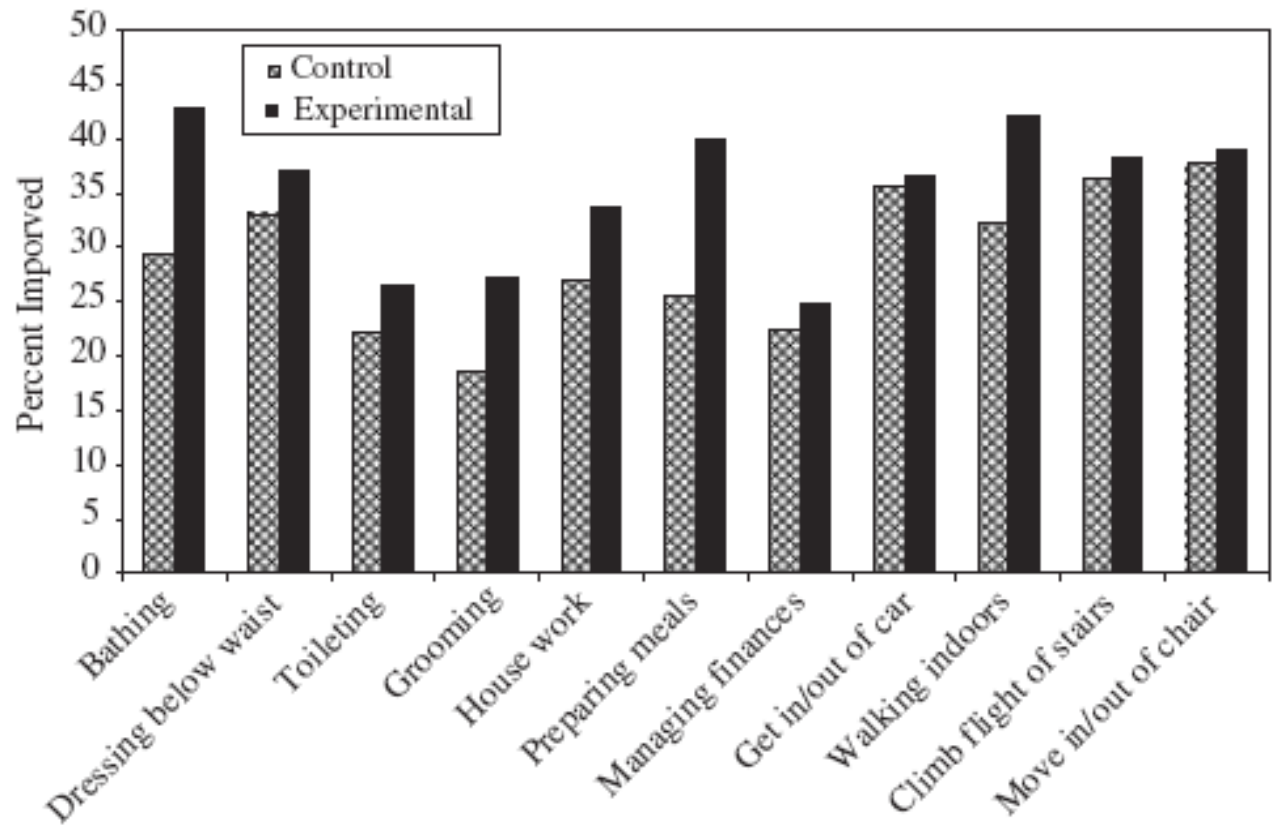

Domains of Functioning

Author's final version prior to publication in Journal of the American Geriatrics Society 54(5):809-816, May 2006. Copyright (C) 2006. The published version is available at http://dx.doi.org/10.1111/j.1532-5415.2006.00703.x 